\title{
Fabrication and Characterization of Anticancer Drug Loaded Double Walled Microspheres
}

\author{
Elangovan Nagarajan*, Dr B Rama, M Swetha, Dr G.S Sharma, Dr L Jyothi Rani, K Sudhamani, \\ Department of pharmaceutics, Malla Reddy Institute of Pharmaceutical Sciences, Maisammaguda, Dhulapally, Secunderabad, Telangana, India.
}

\begin{abstract}
In the present work, double walled microspheres of Tamoxifen (antiestrogenic drug) using Sodium alginate, Hydroxy propyl methyl cellulose (HPMC) K100,Guar gum, Xanthun gum were formulated to deliver Tamoxifen (TMX) through oral route to treat breast cancer patients. Details regarding the preparation and evaluation of the formulations have been discussed in results. From the study following conclusions could be drawn. The results of this investigation indicate that Ion gelation method can be successfully employed to fabricate TMX microspheres. FT-IR spectra of the physical mixture revealed that the drug is compatible with the polymers and copolymer used. Microspheres containing sodium alginate along with HPMC in 1:1 ratio had a least size range of $610 \mu \mathrm{m}$. Increase in the polymer concentration led to increase in \% Yield, \% Drug entrapment efficiency, Particle size. The invitro drug release decreased with increase in the polymer and copolymer concentration. Among all formulations F7 shows Maximum drug release in $12 \mathrm{th} \mathrm{hr}$ when compared with other formulations. Analysis of drug release mechanism showed that the drug release from the formulations followed the Non fickian diffusion mechanism and follows zero order kinectics. Based on the results of evaluation tests formulation coded F7 was concluded as best formulation.
\end{abstract}

Keywords : Tamoxifen, sodium alginate, HPMC, Microspheres, Diffusion, Copolymers, Entrapment efficiency.

Article Info: Received 07 July 2019; $\quad$ Review Completed 14 August 2019; $\quad$ Accepted 18 August 2019; Available online 30 Aug 2019

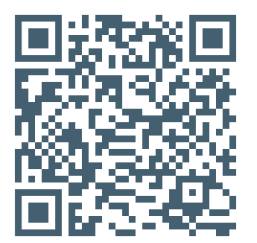

Cite this article as:

Nagarajan E, Rama B, Swetha M, Sharma GS, Jyothi Rani L, Sudhamani K, Fabrication and Characterization of Anticancer Drug Loaded Double Walled Microspheres, Journal of Drug Delivery and Therapeutics. 2019; 9(4-A):79-85 http://dx.doi.org/10.22270/jddt.v9i4-A.3338

*Address for Correspondence:

Elangovan Nagarajan*, Department of pharmaceutics, Malla Reddy Institute of Pharmaceutical Sciences, Maisammaguda, Dhulapally, Secunderabad, Telangana, India.

\section{INTRODUCTION}

For many decades, medication of an acute disease or a chronic illness has been accomplished by delivering drugs to the patients via various pharmaceutical dosage forms like tablets, capsules, pills, creams, ointments, liquids, aerosol, injectables and suppositories as carriers. To achieve and then to maintain the concentration of drug administered within the therapeutically effective range needed for medication, it is often necessary to take this type of drug delivery systems several times a day. This results in a fluctuated drug level and consequently undesirable toxicity and poor efficiency. This factor as well as other factors such as repetitive dosing and unpredictable absorption leads to the concept of controlled drug delivery systems.1,2

The objectives in designing a controlled release system are to deliver the drug at a rate necessary to achieve and maintain a constant drug blood level. This rate should be analogous to that achieved by continuous intravenous infusion where a drug is provided to the patient at a rate just equal to its rate of elimination. This implies that the rate of delivery must be independent of the amount of drug remaining in the dosage form and constant over time. That is release from the dosage form should follow zero-order kinetics. $^{3}$

Micro particles have been widely accepted as a means to achieve oral and parenteral controlled release. The microsphere requires a polymeric substance as a coat material or carrier. A number of different substances biodegradable as well as non- biodegradable have been investigated for the preparation of micro particles 4 . It not only reduces the dose of the drug, reaching to the effective biological sites rapidly but also results in reduced toxicity of the targeting. But in the past few years, pharmacists have been focused their research in colloidal drug delivery system/colloidal carriers, like liposomes, microspheres and nanoparticles as a targeting carriers, which has given selective targeting.

Present microsphere delivery system technology consisting of a single drug dispersed within a polymer matrix has several drawbacks. One is the problem of the so-called "burst effect". By exploiting the phenomenon of phase 
separation between two immiscible polymers dissolved in a mutual solvent, a double-walled microsphere could be manufactured with the second polymer coating the polymer/drug matrix. This one-step process would give a consistent coating of even very small microspheres not achievable via normal, two-step coating processes and would help to smooth out the release curve by lessening the "burst effect" 5 . Along with solving the problem of the "burst effect", this concept of double-walled microspheres could be used to achieve constant release of the drug over long periods of time. So far, this has only been achieved with a limited number of geometric configurations. Since every polymer has its own characteristic release rate, the release could be kept much more constant by changing the polymer type and/or properties. By combining these layers so that the release rate of one layer would complement the slowing of release due to decreased surface area or increased diffusion distances.

Cancer is one of the major health problems in India; Breast cancer is most fatal disease for women worldwide. Tamoxifen (TMX) is a synthetic drug used for breast cancer treatment. TMX acts as antiestrogen agent via active metabolite hydroxytamoxifen. TMX used to treat different stages of breast cancer; in very high doses it may develop some adverse conditions like endometrial cancer and tumor progression. Encapsulation materials were used to minimize the disadvantageous effects and deliver the TMX drug to the tumor site. Nanoparticles, alginate microsphers/microparticles, polymeric micelles and hydrogels are examples for TMX carriers ${ }^{6}$.

\section{MATERIALS AND METHODOLOGY}

\subsection{Materials}

Tamoxifen drug was gifted sample from Chandra labs, Hyderabad. Polymers like HPMC, Sodium alginate, Guar gum and xanthan gum were purchased from S.D. fine chemicals ltd, Mumbai. Other ingredients, solvents and reagents were analytical grade only.

\subsection{Methodology}

\subsubsection{Estimation of Tamoxifen ${ }^{7}$}

Standard Stock solution: $100 \mathrm{mg}$ of Tamoxifen was dissolved in small quantity of Methanol and make up to 100 $\mathrm{ml}$ of $0.1 \mathrm{~N}$ HCL to give a concentration of $(1000 \mu \mathrm{g} / \mathrm{ml})$

Scanning: From the stock solution $100 \mu \mathrm{g} / \mathrm{ml}$ was prepared and UV scan was taken between 200 to $400 \mathrm{~nm}$. The absorption maximum was found to be $239 \mathrm{~nm}$ and was used for the further analytical studies.

\section{Calibration curve of Tamoxifen in 0.1 N HCL:}

The standard solutions were prepared by proper dilutions of the primary stock solution $(1000 \mu \mathrm{g} / \mathrm{ml})$ with buffer to obtain working standards in the concentration range of 5$25 \mu \mathrm{g} / \mathrm{ml}$ of pure sample of Tamoxifen. The concentration of Tamoxifen present in the microspheres was obtained from the calibration curve.

\section{Calibration curve of Tamoxifen in pH6.8 Phosphate buffer:}

Standard Stock solution: $100 \mathrm{mg}$ of Tamoxifen was dissolved in small quantity of ethanol and make up to $100 \mathrm{ml}$ of pH6.8 Phosphate buffer to give a concentration of $(1000 \mu \mathrm{g} / \mathrm{ml})$.

The standard solutions were prepared by proper dilutions of the primary stock solution with buffer to obtain working standards in the concentration range of $5-25 \mu \mathrm{g} / \mathrm{ml}$ of pure sample of Tamoxifen. The concentration of Tamoxifen present in the microspheres was obtained from the calibration curve.

\subsubsection{Drug-Excipients Compatibility study8}

TMX was mixed with all excipients, used in the formulation in different ratios and subjected to FTIR. Prior to the development of the dosage forms the preformulation study was carried out. IR spectral studies lies more in the qualitative identification of substances either in pure form or in combination with polymers and excipients and acts as a tool in establishment of chemical interaction. Since I.R. is related to covalent bonds, the spectra can provide detailed information about the structure of molecular compounds. In order to establish this point, comparisons were made between the spectrum of the substances and the pure compound. The above discussions imply that infrared data is helpful to confirm the identity of the drug and to detect the interaction of the drug with the carriers. FTIR spectra were recorded with a Thermo Nicolet. Japan In the range 400$4000 \mathrm{~cm}-1$ using a resolution of $4 \mathrm{~cm}-1$ and 16 scans. Samples were diluted with $\mathrm{KBr}$ mixing Powder, and pressed to obtain self-supporting disks. Liquid samples formulations were analyzed to form a thin liquid film between two $\mathrm{KBr}$ disks ${ }^{9}$.

\subsubsection{Preparation of double walled microspheres of TMX10,11}

The double walled microspheres were prepared by two step process. In first step the core microspheres of sodium Alginate and with different polymers were formulated. The microspheres then dispersed in the organic phase. The organic phase containing polymer in which drug was dissolved then the organic phase was emulsified with liquid paraffin. The solvent was allowed to evaporate and double walled microspheres were collected.

Formulation of Core Microspheres with Drug: TMX and all other polymers were individually passed through sieve no $\neq 60$. The required quantities of Sodium alginate and the polymer were dissolved in purified water to form a homogenous polymer solution. TMX was added to the polymer solution and mixed thoroughly with a stirrer to form a viscous dispersion. The resulting dispersion was then added manually drop wise into calcium chloride $(1.5 \% \mathrm{w} / \mathrm{v})$ solution through a syringe with a needle of size no.22. The added droplets were retained in the calcium chloride solution for 15 minutes to complete the curing reaction and to produce the spherical rigid microspheres. Microspheres were collected by decantation, and the product thus separated was washed repeatedly with water and dried at $45^{\circ} \mathrm{C}$ for 12 hours.

Formulation of Double Walled Microspheres: The previously formulated microspheres were dispersed in the organic phase. The second polymer 3\%HPMC Phthalate was dissolved in the same organic phase. The resulting organic phase solution was emulsified in liquid paraffin. $1 \%$ span 80 solutions were used as emulsifying agent. Above emulsion was stirred for complete evaporation of the organic solution. After complete evaporation of the organic solution the double walled microspheres were collected by vacuum filtration and washed with $\mathrm{n}$-hexane. The resulted double walled microspheres were freeze dried for $24 \mathrm{hrs}$. 
Table no.1: TMX Double walled Microspheres formulations

\begin{tabular}{|l|l|l|l|l|l|l|l|}
\hline Ingredients (\%) & F1 & F2 & F3 & F4 & F5 & F6 & F7 \\
\hline Tamoxifen (mg) & 100 & 100 & 100 & 100 & 100 & 100 & 100 \\
\hline Sodium alginate (mg) & 200 & 200 & 200 & 200 & 200 & 200 & 200 \\
\hline Guar gum (mg) & - & 100 & - & - & 200 & - & - \\
\hline Xanthun gum & - & - & 100 & - & - & 200 & - \\
\hline HPMC & - & - & - & 100 & - & - & 200 \\
\hline Water(ml) & 20 & 20 & 20 & 20 & 20 & 20 & 20 \\
\hline
\end{tabular}

\begin{tabular}{|l|l|}
\hline Outer phase \\
\hline $\mathrm{CaCl}_{2}$ & $\mathbf{1 g}$ \\
\hline IPA & $\mathbf{5 0 m l}$ \\
\hline
\end{tabular}

\subsubsection{Evaluation of Microspheres ${ }^{12}$}

Scanning electron microscopy (SEM): The morphology of the microspheres was studied using scanning electron microscopy (SEM).The samples for SEM were prepared by lightly sprinkling on a double adhesive tape stuck to an aluminum stub. The stubs were then coated with gold film under reduced pressure. The stub containing the coated samples was placed in the scanning electron microscope (Hitachi S3400N) chamber. The samples were then randomly scanned, and photomicrographs were taken at the acceleration voltage of $5 \mathrm{kV}$. Microphotographs were taken on different magnification and higher magnification was used for surface morphology.

Particle size analysis: Samples of the micro particles were analyzed for particle size by optical microscope. The instrument was calibrated and found that 1unit of eyepiece micrometer was equal to $12.5 \mu \mathrm{m}$. Nearly about 100 Micro particles sizes were calculated under $45 \mathrm{x}$ magnification.

The average particle size was determined by using the Edmo ndson's equation:

$$
D_{\text {mean }}=-\cdots
$$

n

Where,

$\mathrm{n}$ - Number of microspheres observed, $\mathrm{d}$ - Mean size range

Drug Entrapment Efficiency: Microspheres equivalent to $20 \mathrm{mg}$ of the drug were taken for evaluation. The amount of drug entrapped was estimated by crushing the microspheres and extracting with aliquots of pH6.8 Phosphate buffer repeatedly. The extract was transferred to a $100 \mathrm{ml}$ volumetric flask and the volume was made up using pH6.8 Phosphate buffer. The solution was filtered and the absorbance was measured after suitable dilution spectrophotometrically (UV 1700 Shimadzu, Japan) at $239 \mathrm{~nm}$ against appropriate blank. The amount of drug loaded and entrapped in the microspheres was calculated by the following formulas:

\section{Drug entrapment efficiency (\%) = Amount of drug actually present $\times 100$ \\ Theoretical drug load expected}

Determination of Percentage yield: The dried microspheres were weighed and percentage yield of the prepared Microspheres were calculated by using the following formula ${ }^{13}$.

Percentage yield $=\frac{\text { Practical yield }(\mathbf{m g}) \times 100}{\text { Theoretical yield }}$

In-vitro Release Study14: The drug release study was performed for microsphere containing quantity equivalent to $20 \mathrm{mg}$ of TMX Microspheres by using USP dissolution apparatus Type I (Lab India DS 8000) in 900 $\mathrm{ml}$ of $0.1 \mathrm{~N} \mathrm{HCL}$ for first $2 \mathrm{hrs}$ then replaced with pH6.8 Phosphate buffer dissolution media at $50 \mathrm{rpm}$ and $37^{\circ} \mathrm{C}$ temperature. $5 \mathrm{ml}$ of sample was withdrawn at predetermined time interval for 12 hours and same volume of fresh medium was replaced to maintained sink condition Withdrawn samples were assayed spectrophoto metrically at $239 \mathrm{~nm}$. The cumulative percentage drug release was calculated using standard calibration curve.

Release kinetics ${ }^{13}$ : The release data obtained was fitted into various mathematical models. The parameters ' $n$ ', time constant ' $k$ ', the release rate constant ' $R$ ', the regression coefficient were determined by Korsmeyer-peppas equation to understand the release mechanism. To examine the release mechanism of TMX from the microspheres, the release data was fitted into peppa's equation,

$$
\text { Mt } / \mathrm{M} \infty=\mathrm{Kt}^{\mathrm{n}}
$$

Where, Mt/M $\infty$ is the fractional release of drug, ' $t$ ' denotes the release time, ' $\mathrm{K}$ ' represents a constant incorporation structural and geometrical characteristics of the device, ' $n$ ' is the diffusional exponent and characterize the type of release mechanism during the release process.

Table no.2: Diffusional exponent- type of release mechanism

\begin{tabular}{|c|c|}
\hline $\begin{array}{c}\text { Release exponent } \\
\text { (n) }\end{array}$ & $\begin{array}{c}\text { Drug transport } \\
\text { mechanism }\end{array}$ \\
\hline 0.5 & Fickian diffusion \\
\hline $0.5<\mathrm{n}<1.0$ & $\begin{array}{c}\text { Anomalous transport or } \\
\text { non-Fickian }\end{array}$ \\
\hline 1.0 & Case-II transport \\
\hline Higher than 1.0 & Super Case-II transport \\
\hline
\end{tabular}


If $\mathrm{n}<0.5$, the polymer relaxation does not affect the molecular transport,

hence diffussion is Fickian.

If $\mathrm{n}>0.5$, the solid transport will be non-fickian and will be relaxation controlled.

Other equations to study the drug release kinetics from dosage forms

\section{a. Zero order}

\section{$\% \mathbf{R}=\mathbf{k t}$}

This model represents an ideal release in order to achieve prolonged pharmacological action. This is applicable to dosage froms like transdermal systems, coated forms, osmotic systems, as well as Matrix tablets containing low soluble drugs.

\section{b. First order}

$\log$ (fraction unreleased) $=\mathrm{kt} / 2.303$

The model is applicable to hydrolysis kinetics and to study the release profiles of pharmaceutical dosage forms such as those containing water soluble drugs in porous matrices.

\section{c. Matrix (Higuchi Matrix)}

\section{$\% \mathrm{R}=\mathrm{kt}^{0.5}$}

This model is applicable to systems with drug dispersed in uniform swellable polymer matrix as in case of matrix tablets with water soluble drug.

\section{d. Peppas Korsmeyer Equation}

$$
\begin{gathered}
\% \mathrm{R}=\mathrm{kt}^{\mathrm{n}} \\
\log \% \mathrm{R}=\operatorname{logk}+\text { nlogt }
\end{gathered}
$$

This model is widely used when release mechanism is well known or when more than one type of release phenomenon could be involved.

\section{RESULTS AND DISCUSSION}

\subsection{Estimation of Tamoxifen (API)}

Table no.3: Description, solubility and melting point of TMX (API)

\begin{tabular}{|l|l|}
\hline Description & $\begin{array}{l}\text { Color - A white or almost white crystalline powder } \\
\text { Odor - Free of odor }\end{array}$ \\
\hline Solubility & $\begin{array}{l}\text { Methanol - Freely soluble } \\
\text { Dichloromethane - Soluble } \\
\text { Water - Slightly soluble } \\
\text { Acetonitrile - Practically soluble }\end{array}$ \\
\hline Mpt & $192-195^{\circ} \mathrm{C}$ \\
\hline
\end{tabular}

\subsection{Scanning:}

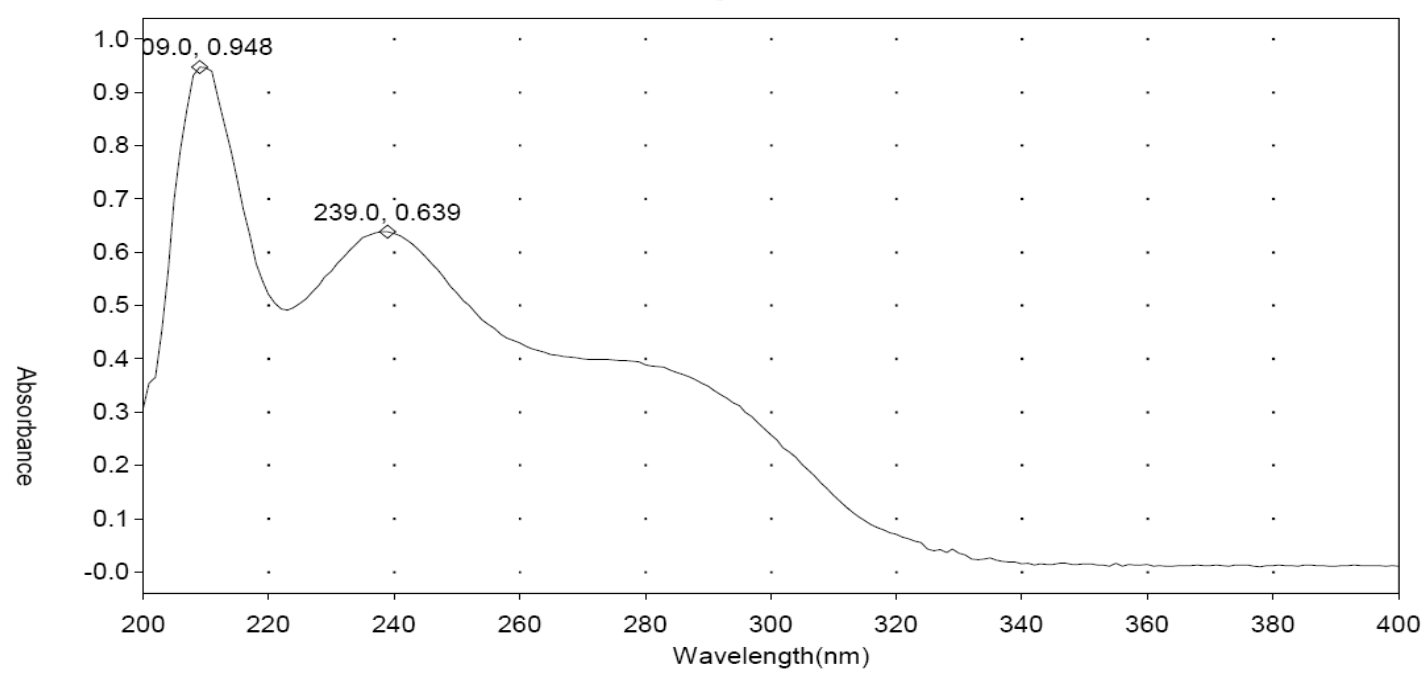

Figure no.1: UV Visible spectrum of TMX observed at 239nm 
3.3 Calibration curve of Tamoxifen in 0.1N HCL and 6.8pH phosphate buffer:
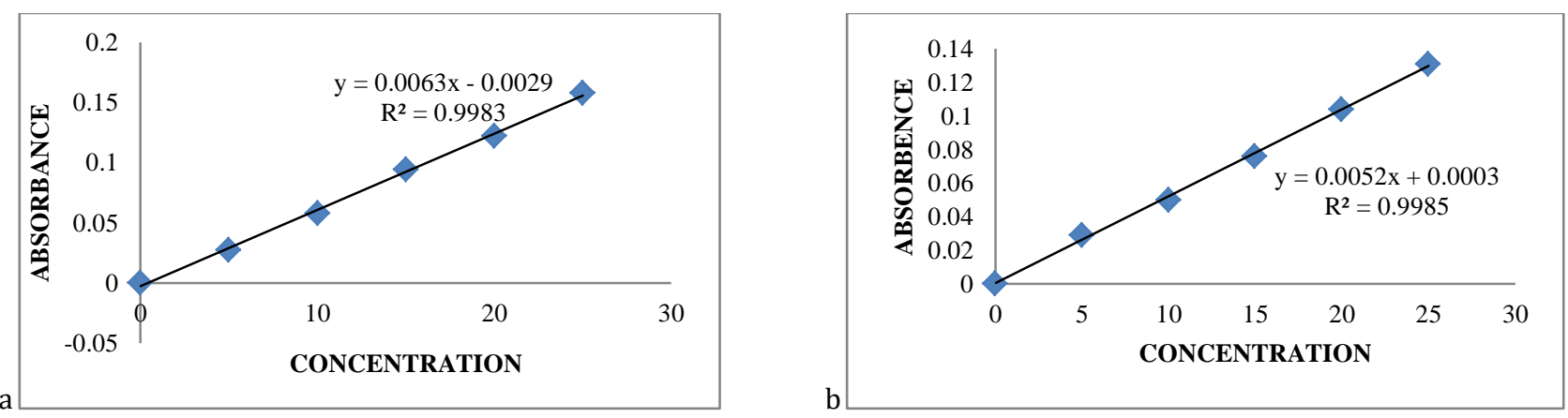

Figure no.2: Linearity curve of TMX in a) 0.1N HCL b) 6.8pH phosphate buffer

3.4 Drug and Excipients compatibility studies (FTIR):

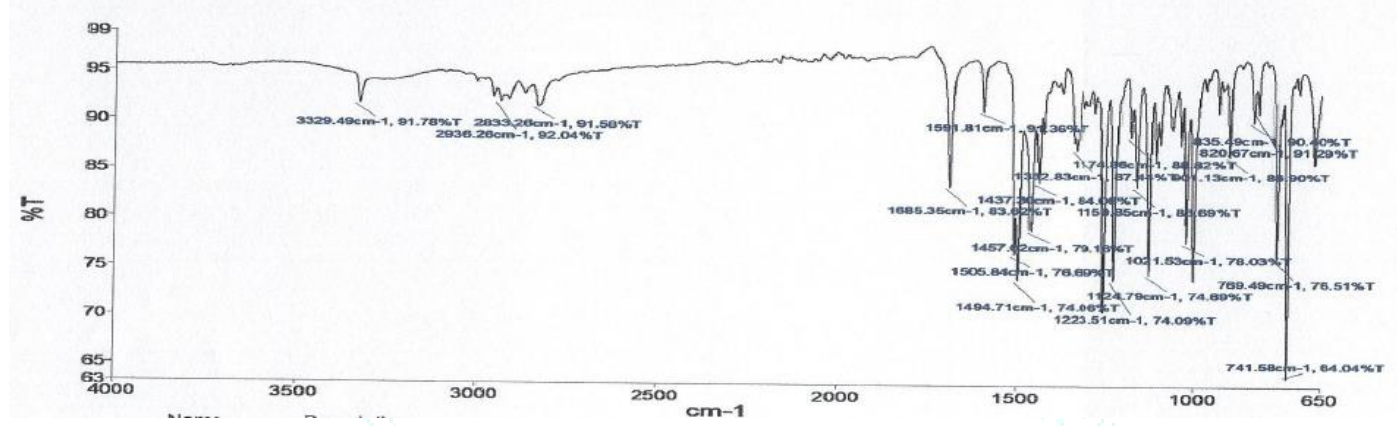

Figure no.3: FTIR spectra of TMX (API)

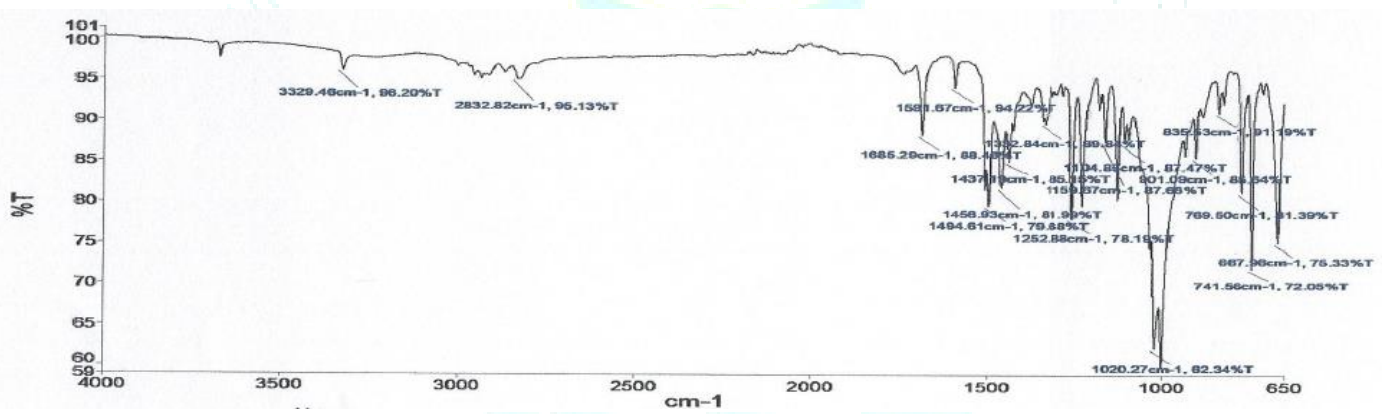

Figure no.4: FTIR Spectra of TMX optimized formulation

Table no.4: FTIR Interpretation Table

\begin{tabular}{|l|l|l|l|}
\hline Characteristic peak & Observed range & Pure drug & Optimized formulation \\
\hline C-H STRECH & $300-2830$ & 2833.26 & 2832.82 \\
\hline C-N & $1260-1020$ & 1223.51 & 1252.88 \\
\hline
\end{tabular}

FTIR studies were performed to understand the compatibilities between the drugs with different excipients. The figures above illustrate that the functional groups like C$\mathrm{H}$ STRECH with the observation range of 300-2830 has peaks at 2833.26 in pure drug and 2832.82 in optimized formulation. Similarly the functional group C-N has a peak range of 1260-1020 has peaks at 1223.51 in pure drug and 1252.88 in optimized formulation. The functional groups in both the pure drug and optimized formulation are found Hence it can be concluded that the pure drug is compatible with the excipients used in the study.

\subsection{Evaluation and characterization of Microspheres:}

\subsubsection{Percentage Yield and Drug entrapment efficiency:}

Percentage yield was found to be in the range of 79.7 to $86.4 \%$ for microspheres containing sodium alginate along with other polymers.
Percentage Drug entrapment efficiency of TMX in different formulations ranged from 76.5 to $89.4 \%$ for double walled microspheres. Drug entrapment efficiency increases with the increase in proportion of the respective polymers concentration, particle size also increases exponentially with increased viscosity of dispersed phase.

Table no.5: Percentage yield and Drug entrapment efficiency of TMX microspheres

\begin{tabular}{|c|l|l|}
\hline $\begin{array}{l}\text { Formulation } \\
\text { code }\end{array}$ & $\begin{array}{l}\text { Percentage } \\
\text { yield }\end{array}$ & $\begin{array}{l}\text { \%Drug entrapment } \\
\text { efficiency }\end{array}$ \\
\hline F1 & 81.1 & 80.2 \\
\hline F2 & 84.6 & 76.5 \\
\hline F3 & 85.8 & 84.7 \\
\hline F4 & 86.4 & 85.2 \\
\hline F5 & 79.7 & 86.6 \\
\hline F6 & 84.2 & 88.2 \\
\hline F7 & 85.9 & 89.4 \\
\hline
\end{tabular}


3.5.2 Particle size analysis: The mean size increased with increasing polymer concentration which is due to a significant increase in the viscosity, leads to increase in droplet size and finally a higher microspheres size. Microspheres containing least polymer concentration had achieved lease size range of $610 \mu \mathrm{m}$.

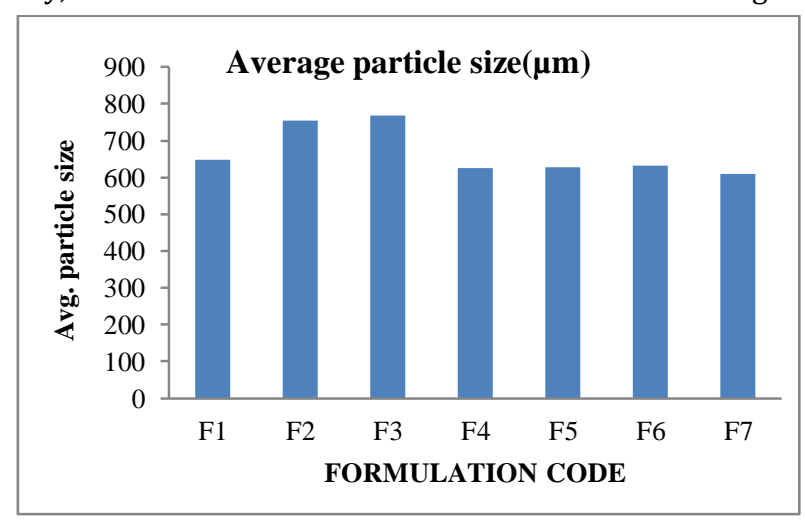

Figure no.5: Average particle size of TMX microspheres

\subsubsection{Scanning Electron Microscopy (SEM):}
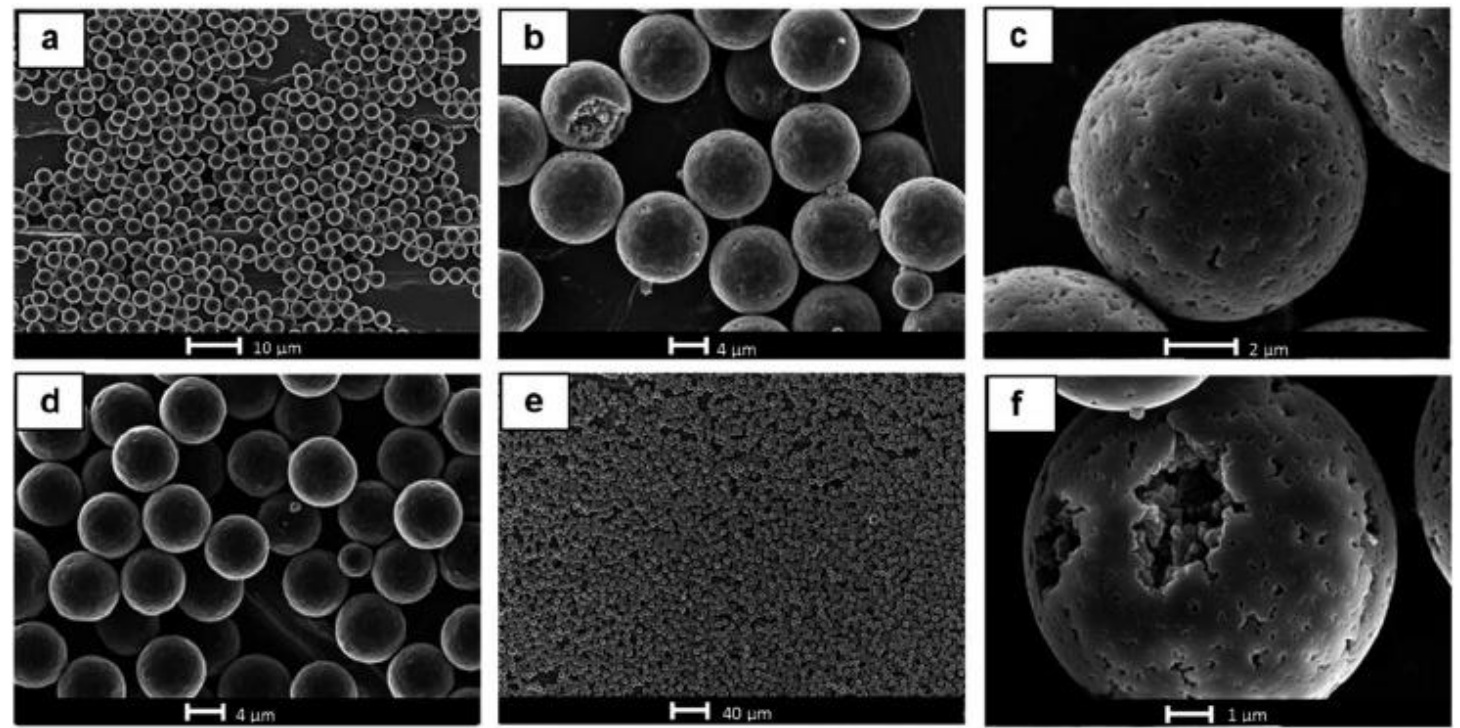

Figure no.6: SEM of a,e) solid microspheres; c) surface morphology; b,d) microspheres of varying size; f) perforated microspheres.

\subsubsection{In-vitro drug release studies}

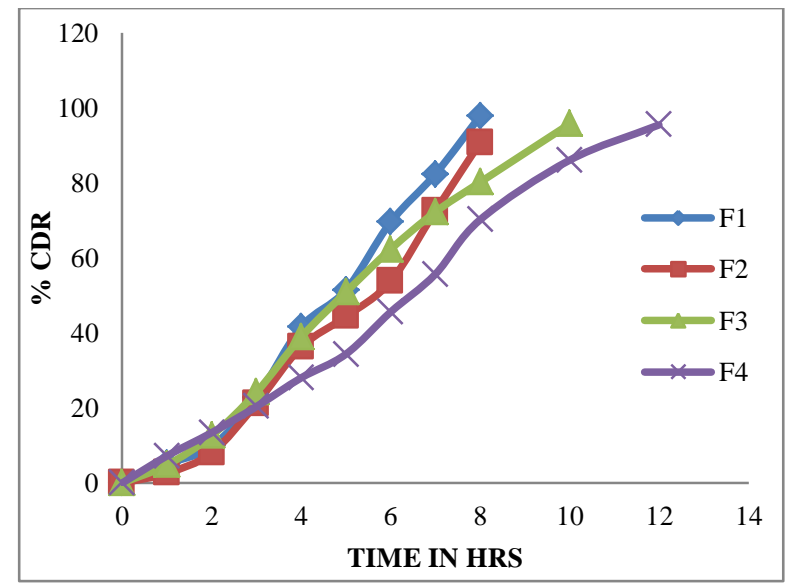

Figure no.7: In-vitro drug release profile of TMX Microspheres

(F1-97.74/8hrs, F2-90.64/8hrs, F3-92.62/10hrs, F495.4/12hrs)

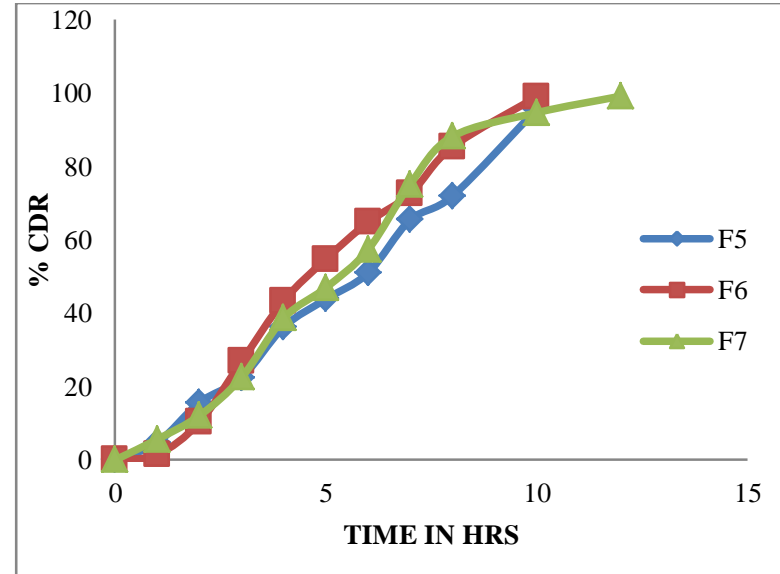

Figure no.8: In-vitro drug release profile of TMX Microspheres

(F5-94.51/10hrs, F6-95.84/10hrs, F7-99.1/12hrs) 
As per the dissoultution study of formulations F1-F7, the maximum drug release is observed for F7 formulation at $12^{\text {th }}$ hour, hence F7 considered as optimized formulation.

\section{Drug Release Kinetics:}

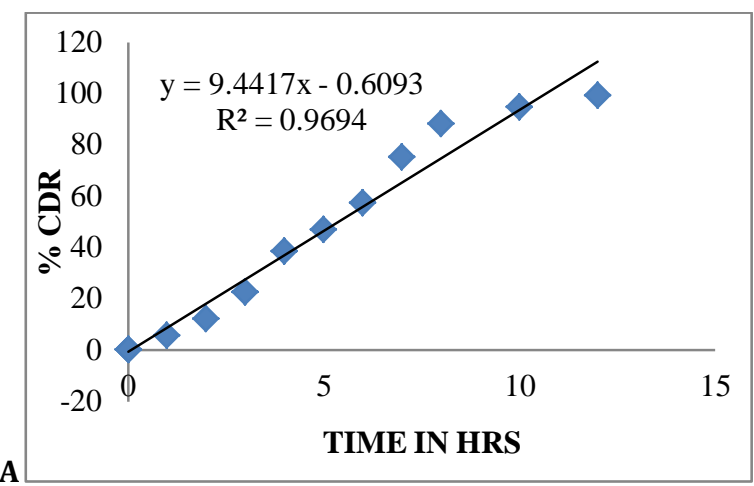

A
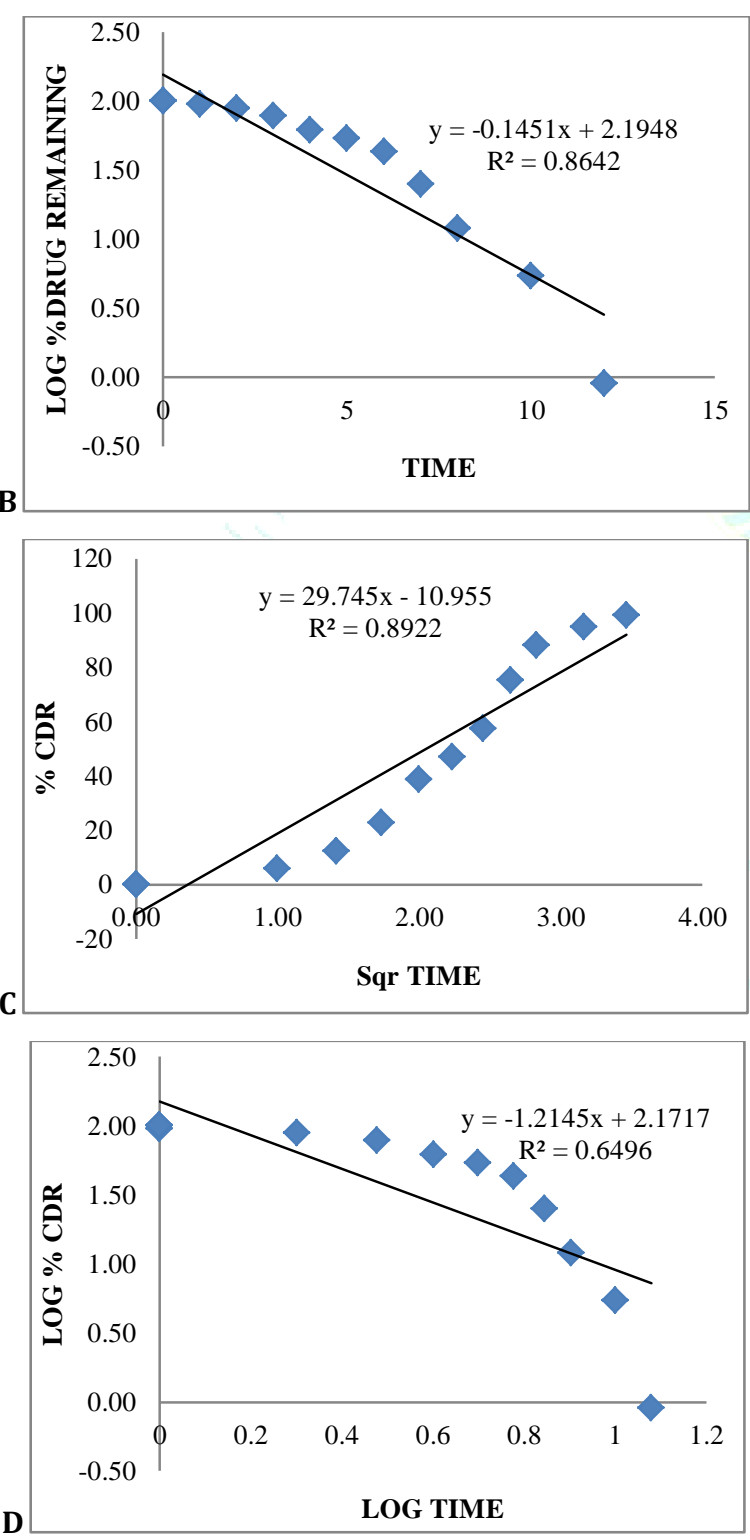

Figure no.9: Drug release kinetics for TMX F7 formulation: A) Zero order, B) First order, C) Higuchi and D) Korsmeyer-Peppas plot
Based on coefficient of determination and release exponent values, it can be suggested that the mechanism of drug release follows Zero order kinetics which is independent on concentration and Peppas model shows Non-fickian diffusion mechanism. In-vitro drug release of TMX microspheres followed combination of diffusion and spheres erosion.

\section{CONCLUSION}

In present work, double walled microspheres of TMX were fabricated by Ion gelation method to deliver TMX via oral route using Sodium alginate, HPMC, Guar gum, and Xanthun gum polymers. Increase in the polymer concentration lead to increase in percentage yield, drug entrapment efficiency and particle size. Optimized F7 formulation followed Zero order and Non-fickian diffusion mechanism.

\section{REFERENCES}

1. Mohammed G Ahmed, Satish K BP, Kiran K GB, Formulation and Evaluation of Gastric-Mucoadhesive Drug Delivery Systems of Captopril, JCPR, 2010; 2(1): 26-32.

2. Hannah B, Novel bioadhesive formulation in drug delivery, The drug delivery companies report. (2004); 16-19.

3. Mathiowitz E, Chickering DE, Jacob JS, Biologically erodible microspheres as potential oral drug delivery system, 1997; 386(6623): 410

4. Sudhakar Y, Kuotsu K, BandyopadhyayAK, Buccal bioadhesive drug delivery - a promising option for orally less efficient drugs. J. Control. Release. 2006; 114: 15-40.

5. ekarek KJ, Jacob JS and Mathiowitz E. One-step preparation of double walled Microspheres. Adv Mater. 1994; 6(9):684- 687.

6. Onur Korkmaz, Bernis Girgin, etc, Production and investigation of controlled drug release properties of Tamoxifen loaded alginate-gum Arabic microbeads. Journal of the Turkish chemical society, 2016; 3(3): 47-58.

7. M.L Thakkar, D.B Shah and D.G Maheshwari, Simultaneous spectroscopic estimation and validation of Gemcitabine and Tamoxifen in synthetic mixture by first order derivative method, International journal of pharmaceutical sciences and research, 2015; 6(9):4000-03.

8. Priyanka patel, Kajal Ahir, Vandana Patel, Lata Manani, Chirag patel, Drug-Excipient compatability studies:First step for dosage form development, The pharma innovation journal, 2015; 4(5): 14-20.

9. Wen-Hsuan chiang, Viet Thang Ho, Et.al, Dual stimuliResponsive polymeric Hollow Nanogels Designed as carriers for intracellular triggered drug release, ACS publications, 2012; 28(42): 15056-15064.

10. Mahale Manisha M, Saudagar R B, Microshpere: A Review, Journal of Drug Delivery and Therapeutics, 2019; 9(3-S): 854856.

11. A Kishore Babu, N Bhanu Teja, B Ramakrishna, B Balagangadhar, B Vijay kumar and G Venkata reddy, Formulation and Evaluation of Double walled microspheres loaded with Pantoprazole, IJRPC, 2011; 1(4): 770-779.

12. Gopfe rich A, Alonso MJ and Langer R, Development and characterization of microencapsulated microspheres, Pharm Research.1994; 11(11):1568- 1574.

13. Venkateswara murthy.N, Sambathkumar, Clarithromycin mucoadhesive microspheres for anti-helicobacter Pylori therapy, Formulation and in vitro evaluation, International Journal of Current Pharmaceutical Research, 2010; 2(3):3641.

14. Twarita Deshpande, Rajashree Masareddy, Development of mucoadhesive microspheres for nasal delivery of sumatriptan, International Journal of Pharmaceutical Sciences Review and Research. 2011; 7(2):25-31. 